INVESTIGACIONES

\title{
HABILIDADES COMUNICATIVAS EN L2 MEDIATIZADAS POR LA TECNOLOGIA EN EL CONTEXTO DE LOS ENFOQUES POR TAREAS Y COOPERATIVO
}

\author{
COMMUNiCATIVE ABILITIES IN L2 MEDIATED BY TECHNOLOGY IN THE CONTEXT \\ OF TASK BASED TEACHING AND COOPERATIVE LEARNING
}

\author{
Habilidades comunicativas em L2 mediatizadas pela tecnologia no contexto \\ dos enfoques por tarefas e cooperativo
}

\author{
Angie Quintanilla Espinoza ${ }^{1}$, Anita Ferreira Cabrera ${ }^{2}$ \\ ${ }^{1}$ Universidad de Concepción, Chile, 95467721 anquinta@udec.cl \\ ${ }^{2}$ Universidad de Concepción, Chile, aferreir@udec.cl
}

\begin{abstract}
RESUMEN
El objetivo principal de este artículo es determinar cómo los principios metodológicos del enfoque basado en tareas, el aprendizaje cooperativo y la metodología CALL pueden ser utilizados de manera combinada en el diseño de actividades para el desarrollo de las habilidades lingüísticas. Con este objetivo, se explora evidencia empírica acerca de la efectividad de esta metodología en el aprendizaje de inglés como lengua extranjera en un estudio cuasiexperimental pre- y post- test grupo control. Los resultados obtenidos revelan un mayor incremento en el aprendizaje del grupo experimental que utilizó la metodología integrada en comparación con el grupo control que trabajó con una metodología tradicional. Esto sugiere que el uso combinado del enfoque basado en tareas, el aprendizaje cooperativo y la metodología CALL sería beneficioso para el desarrollo de las habilidades lingüísticas.
\end{abstract}

Palabras clave: adquisición de segundas lenguas, aprendizaje de lenguas asistido por computador, enseñanza de lenguas basada en tareas, aprendizaje cooperativo.

\begin{abstract}
The main objective of this article is to portray how the theoretical principles of task-based teaching, cooperative learning and CALL can be used together in the design of activities for the improvement of linguistic abilities. To achieve this, empirical evidence was examined in order to determine the efficiency of this integrated methodology vs. the traditional one commonly used for teaching English as a foreign language. Both methods were contrasted and tested in an experimental pre/post test control group research design. The results show that the experimental group, which used the integrated methodology, had a considerable language improvement compared to the control group that used a traditional one. This suggests that an integrated methodology using the approach of the task-based teaching, the cooperative learning and CALL would be beneficial for the improvement of linguistic abilities.

Key words: second language acquisition, computer assisted language learning, task based language teaching, cooperative learning.
\end{abstract}

\section{RESUMO}

O objetivo principal deste artigo é determinar como os princípios teóricos do enfoque baseado em tarefas, a aprendizagem cooperativa e a metodologia CALL podem ser utilizados de maneira combinada no desenho de atividades para a melhora das habilidades lingüísticas. Com este objetivo, explora-se a evidência empírica a respeito da efetividade desta metodologia na aprendizagem do inglês como língua estrangeira num estudo cuasiexperimental com um pré- e pós-teste e um grupo controle. Os resultados obtidos revelam um maior incremento na aprendizagem do grupo experimental que utilizou a metodologia integrada em comparação com o grupo controle que trabalhou com uma metodologia tradicional. Isto sugere que o uso combinado do enfoque baseado em tarefas, a aprendizagem cooperativa e a metodologia CALL seria benéfico para o desenvolvimento das habilidades lingüísticas.

Palavras-chave: aquisição de segundas línguas, aprendizagem de línguas assistido por computador, ensino de línguas baseado em tarefas, aprendizagem cooperativa.

\footnotetext{
* *Proyecto FONDECYT 1068185.
} 


\section{INTRODUCCION}

Durante las pasadas dos décadas la metodología de enseñanza de las lenguas extranjeras ha estado caracterizada por un claro cambio de enfoque: de la enseñanza centrada en el profesor, en los contenidos y en el método se ha pasado a la enseñanza centrada en el alumno (en sus intereses y necesidades). En esta enseñanza centrada en el alumno, el profesor debe detectar las falencias y/o necesidades de los estudiantes. Algunas de las mayores dificultades en relación con el aprendizaje de lenguas son: la poca exposición que tienen los alumnos a la lengua meta, el excesivo trabajo en las formas gramaticales y el predominio de las habilidades lingüísticas de comprensión oral y escrita en el trabajo en el aula, lo que por consiguiente circunscribe al estudiante a un rol más bien receptivo. A partir de este rol receptivo, el estudiante utiliza la lengua, de forma oral o escrita, en contextos reducidos y poco significativos.

El estudio propuesto en este artículo se enmarca en el ámbito de la Lingüística Aplicada, específicamente en la enseñanza de Segundas Lenguas, a partir de la creación de un módulo de intervención lingüística que utiliza una metodología "mixta" basada en Enfoque Basado en Tareas y el Enfoque Cooperativo, además de incorporar la aplicación de CALL a través del uso de E-mail, Weblogs e Internet como herramientas de aprendizaje. Esto surge a partir de la necesidad de introducir metodologías de enseñanza-aprendizaje eficientes en ambientes de aprendizaje mediatizados por la tecnología. El objetivo es desarrollar e implementar una metodología que incremente la habilidad comunicativa en inglés como lengua extranjera, a partir del uso de CALL, el enfoque por tareas y cooperativo y obtener evidencia empírica de la efectividad de la metodología propuesta.

El Aprendizaje de Lenguas Asistido por Computador (del inglés, Computer Assisted Language Learning (CALL)) a su vez responde a la necesidad de incorporar la tecnología en las sala de clases, puesto que los alumnos están insertos en una sociedad tecnológica donde los celulares, mp3, mp4, I-pod e internet son utilizados con el propósito de comunicarse, obtener y/o almacenar información. En este contexto, CALL se dedica a explorar el papel de estas tecnologías en el proceso de enseñanza-aprendizaje de lenguas. $\mathrm{Su}$ objetivo es mejorar el aprendizaje en aquellos que aprenden un idioma a través de medios computacionales, permitiendo que el aprendiz desarrolle, expanda y refine sus habilidades comunicacionales en una nueva lengua.

Uno de los aspectos fundamentales de la metodología de CALL es determinar el enfoque de enseñanza que orienta el diseño y desarrollo de una aplicación computacional para la enseñanza de la lengua. En este contexto, los enfoques por tareas y cooperativo han resultado ser efectivos (Morales y Ferreira, 2008). El enfoque por tareas tiene como objetivo hacer participar a los estudiantes en la resolución de tareas significativas con objetivos comunicativos útiles y reales, las cuales favorecen el protagonismo del estudiante y le brindan la oportunidad de utilizar los conocimientos que posee para solucionar los retos que le impone la tarea centrando la resolución de la misma en el significado más que en la forma (Willis, 1996; Skehan, 1996; Richards y Rodgers, 2001). El enfoque cooperativo (Johnson y Johnson, 1987; Slavin, 1990; Sharan y Sharan, 1992; Walters, 2000) en la búsqueda de una mayor y más rica interacción en el aula enfatiza técnicas de trabajo cooperativo en las cuales los alumnos deben trabajar conjuntamente de forma interdependiente y coordinada con los otros miembros del grupo, desarrollando en los estudiantes competencias lingüísticas, sociales y cognitivas a través de la interacción y el 
trabajo en equipo. También, promueve una atmósfera positiva, la cual es necesaria para una interacción satisfactoria en el aula. En este contexto, se incorporan las tecnologías de información y comunicación a través del uso de páginas de internet, correo electrónico y weblog, apoyados en la metodología CALL, específicamente CMC, la cual señala que el uso de la comunicación mediada por computadores puede favorecer el desarrollo de las habilidades comunicativas.

El artículo está organizado en las siguientes secciones: En la sección 1 se incluyen las metodologías para la enseñanza de lenguas incluidas en esta investigación En la sección 2 se expone la metodología CALL. En la sección 3 se presentan investigaciones empíricas que se han llevado a cabo en algunas de las áreas temáticas presentes en esta investigación. En la sección 4 se describe el estudio cuasiexperimental longitudinal, se delimita la metodología de investigación y se describe el módulo de intervención lingüística. En la sección 5 se presentan los resultados del estudio. Finalmente, en la sección 6 se presentan las conclusiones.

\section{METODOLOGIAS PARA LA ENSEÑANZA DE LENGUAS}

\subsection{LA ENSEÑANZA DE LENGUAS BASADA EN TAREAS}

El Enfoque Basado en Tareas surge como una propuesta al interior del enfoque comunicativo de enseñanza de lenguas. Aquí el desarrollo de la competencia comunicativa de los estudiantes depende de que el alumno sea un agente activo de su propio aprendizaje, mientras que el profesor actúa como mediador en este proceso.

En la literatura especializada no existe un acuerdo en relación con qué constituye una tarea, lo que hace que la definición de tarea sea problemática. Es posible encontrar definiciones de tarea comunicativa elaboradas por diversos autores e investigadores (Long, 1985; Prabhu, 1987; Nunan, 1989; Willis, 1996; Bygate, Skehan, y Swain 2001, entre otros). En términos generales, una tarea puede ser definida como un plan de trabajo cuyo objetivo es que el estudiante use la lengua enfocándose en el significado haciendo uso de cualquiera de las cuatro habilidades o de combinaciones de éstas con la finalidad de lograr una meta relacionada con el mundo real (un resultado no-lingüístico) a través de procesos cognitivos tales como seleccionar, ordenar y razonar.

Estas tareas han sido clasificadas por distintos autores siguiendo distintos criterios, como son el nivel de dificultad, los objetivos que persiguen, ya sean cognitivos o comunicativos, Prahbu (1987) propone una clasificación de acuerdo a las operaciones cognitivas que debe realizar el alumno durante la resolución de la tareas, autores como Pica, Kanagy y Flodum (1993) proponen una clasificación psicolingüística, la cual ordena las tareas según el potencial de éstas en el proceso de aprendizaje de la lengua. Del mismo modo, Willis (1996) propone una clasificación denominada pedagógica (una de las más nombradas en la literatura) que clasifica las tareas según el tipo de operaciones que los alumnos deben llevar a cabo en ellas. Algunas de estas operaciones son: hacer listas, ordenar y clasificar, comparar, resolver problemas, compartir experiencias personales y las denominadas tareas creativas. Además de estas clasificaciones, Estaire (2004) propone una clasificación de acuerdo a la función que cumple la tarea dentro de un curso de lengua, por lo cual diferencia entre tareas de comunicación (centradas en el significado) y tareas de apoyo lingüístico (centradas en los contenidos lingüísticos). 
Durante el desarrollo de estas tareas, se deben considerar tres fases bien definidas: la primera fase, pretarea que involucra las actividades que deben realizar el profesor y los estudiantes antes de comenzar la tarea con el objetivo de preparar a los estudiantes para realizar la tarea de tal modo que ésta promueva la adquisición. La segunda fase, durante la tarea, se centra en la tarea misma, aquí se desarrollan principalmente actividades de comprensión, expresión o interacción. En esta fase la intervención del profesor es menor y predomina la interacción entre los alumnos, propiciando el uso de la lengua extranjera en función la tarea. Finalmente, la fase de posttarea involucra procedimientos de seguimiento mediante los cuales el alumno puede monitorear su aprendizaje y el logro del objetivo final de la tarea, además de reflexionar en la tarea y promover la atención en la forma, particularmente en las que resultaron problemáticas durante el desarrollo de la tarea.

Las tareas pueden ser utilizadas como unidad central de planificación de unidades didácticas. Así, Estaire (2004) señala que existen distintas formas de organizar una unidad mediante tareas, pero propone seis fases en la programación de unidades didácticas basadas en tareas; a su vez, señala que estas fases han probado ser un instrumento eficaz para integrar los diferentes ejes del proceso educativo: objetivos, contenidos, metodología y evaluación. En estas fases se debe determinar: (1) el tema de la unidad, (2) los objetivos, (3) la tarea que realizarán los alumnos al final de la unidad, (4) los contenidos lingüísticos necesarios para la consecución de la tarea final, (5) la secuencia de tareas que realizarán los alumnos en las clases previas a la tarea final, (6) los instrumentos y procedimientos de evaluación. Considerar estas fases en la programación de las unidades didácticas permitirá el uso efectivo del enfoque por tareas y la correcta consecución de las tareas finales.

\subsection{EL APRENDIZAJE COOPERATIVO PARA LA ENSEÑANZA DE LENGUAS}

El aprendizaje cooperativo (Johnson y Johnson, 1987; Slavin, 1990) nace como una forma de responder a la problemática de la competitividad en las salas de clases y a la vez aumentar la posibilidad de refuerzo y la motivación de los estudiantes más débiles. Si bien el aprendizaje cooperativo se desarrolló originalmente para la educación general, su uso se ha expandido a la enseñanza-aprendizaje de segundas lenguas.

El concepto de aprendizaje cooperativo comprende un conjunto de diferentes técnicas y métodos que a veces tienen poco en común y suelen diferenciarse en cuanto al grado de interdependencia de las recompensas, grado de interdependencia de la tarea, grado de responsabilidad individual, grado de estructura impuesta por el profesor o por la propia tarea y grado de utilización de la competición que puede llegar hasta la no competición.

De acuerdo a Slavin (1990) existen tres elementos esenciales en un programa de aprendizaje cooperativo para que este sea exitoso: establecer una meta para todo el grupo, la cual los motivará a ayudarse mutuamente; considerar que el éxito del grupo se basa en el aprendizaje individual de cada uno de los miembros y, finalmente, reconocer que los estudiantes contribuyen a su equipo mejorando sus actuaciones pasadas.

El Aprendizaje Cooperativo intenta redefinir los roles del profesor y del alumno a través de una metodología que depende menos de una enseñanza dirigida por el profesor y se centra en un trabajo cooperativo en grupo o en parejas. De acuerdo a Johnson y Johnson (1998) el docente cumple seis roles en el aprendizaje cooperativo: (1) especifica 
los objetivos de la actividad, (2) toma decisiones previas a la enseñanza respecto de la dinámica de trabajo, (3) explica la tarea y estructura los objetivos de las tutorías, (4) pone en marcha la actividad cooperativa, (5) controla la efectividad del trabajo cooperativo, e interviene cuando es necesario y (6) evalúa los logros de los alumnos y los ayuda a discutir cómo ha resultado la colaboración en el curso. Por lo tanto, el papel del profesor en relación con el trabajo en grupo determina por una parte el buen funcionamiento del grupo y la consecución de los objetivos y, por otra, el bienestar de todos los alumnos que participan en el grupo.

A su vez, el rol del alumno también sufre modificaciones. El aprendizaje cooperativo demanda que el alumno tome un rol más activo y que se responsabilice por su propio aprendizaje. Y a su vez, que aprenda a explicar, comprometerse, negociar y motivar a los demás, cuando participa como miembro del grupo. Dentro de los grupos es importante asignar roles a cada uno de los miembros y especificar el papel que cada uno debe asumir y la manera de ejecutarlo, a fin de conseguir efectos positivos sobre el rendimiento y resguardar que cada uno de los estudiantes asuma una carga mental menor y que se distribuya la responsabilidad y el esfuerzo. Algunos de los roles que pueden asignarse dentro de un grupo (Willis y Willis 2007) son: escritor o secretario, portavoz, consultor de lenguaje, líder, observador (estos roles pueden modificarse en grupos más pequeños).

En relación con el aprendizaje cooperativo, Walters (2000) señala cuatro modelos principales: Jigsaw, Aprendizaje por Equipos, Aprendiendo Juntos e Investigación de Grupo. Las diferencias entre ellos se encuentran en el grado de estructuración de la tarea, la utilización de recompensas, la competición y los métodos de evaluación individual.

\section{METODOLOGIA CALL}

El Aprendizaje de Lenguas Asistido por Computador (del inglés, Computer Assisted Language Learning (CALL)) es un campo académico que se dedica a explorar el papel de las tecnologías de la información y de la comunicación en el aprendizaje y la enseñanza de lenguas. De acuerdo a Cameron (1999), el objetivo principal de CALL es "mejorar la capacidad de aprendizaje de aquellos que aprenden un idioma a través de medios computacionales". Si bien los orígenes de CALL pueden situarse en los años 60 , ha sido en las últimas décadas que la investigación ha volcado su interés en esta creciente disciplina, lo que ha sido de alguna manera impulsado por el continuo avance tecnológico y las múltiples posibilidades que CALL ofrece.

\subsection{TECNOLOGIAS DE LA INFORMACION Y COMUNICACION (TICS)}

Desde la aparición de Internet en la década de los 90 World Wide Web (WWW) se ha convertido en una herramienta de fácil acceso a distintos tipos de información, y no sólo ha facilitado el acceso a la información sino que ha mejorado los canales de comunicación permitiendo que ésta sea más rápida y eficaz con personas de todo el mundo, y en el caso de la enseñanza de lenguas permite el intercambio en la lengua de estudio.

El uso de TICs permite un aprendizaje de las lenguas de tipo constructivista, el cual está centrado en el estudiante e integra las destrezas comunicativas con la posibilidad de disponer de actividades para distintos niveles de competencia en el aula. En relación 
con la enseñanza y aprendizaje de lenguas, es posible señalar algunas de las ventajas de la red:

- Favorece la integración de las destrezas lingüísticas, dada su capacidad multimedia.

- Ofrece contextualización de las muestras de lengua y permite el acceso a todo tipo de input lingüístico con materiales auténticos y actuales.

- Facilita la interacción comunicativa y la negociación de significados.

- Potencia la autonomía del alumno que irá marcando el camino a seguir en la búsqueda de la información.

- Potencia la interculturalidad, puesto que posibilita que el alumno experimente con la lengua que está aprendiendo en un contexto sociocultural real y variado.

- Aumenta la motivación de los alumnos debido a las amplias posibilidades de comunicación, a los materiales atractivos sensorialmente, la flexibilidad y la novedad.

\subsection{COMUNICACION MEDIADA POR COMPUTADOR (CMC)}

La Comunicación Mediada por Computador, o CMC, en términos simples puede definirse como cualquier tipo de comunicación humana lograda a través de o con la ayuda de computadores. Esta comunicación puede ser de tipo sincrónica, es decir, la comunicación ocurre en tiempo real, o asincrónica, es decir, la comunicación no es en tiempo real. La comunicación resultante del uso de CMC es única y posee características tanto del discurso escrito como del hablado. Uno de los tantos beneficios de CMC es que los intercambios en red parecen ayudar a todos los individuos en clases de idiomas a participar de manera más frecuente, con mayor confianza y con mayor entusiasmo en el proceso comunicativo, que lo que los mismos estudiantes harían en clases orales.

Correo Electrónico o E-mail: como medio de comunicación asincrónico ofrece a los estudiantes la posibilidad de entablar comunicación real a través del intercambio de mensajes con otros. Algunos de los beneficios que posee el E-mail como herramienta para la enseñanza de lenguas son que permite extender el tiempo y lugar de aprendizaje y que a la vez provee las mismas posibilidades de interacción para todos los estudiantes.

Weblog: también de tipo asincrónico, es un sitio web que se actualiza periódicamente y que recopila textos, artículos o comentarios de interés sobre diversos temas, también puede incluir enlaces web, imágenes y videos. Algunas de sus ventajas para la enseñanza de lenguas son las siguientes: favorece la interacción social, favorece el aprendizaje cooperativo, promueve el uso de nuevas tecnologías, favorece la participación de los estudiantes, aumenta la motivación, favorece la habilidad escrita y lectora y une el proceso de aprendizaje con el mundo real.

\section{INVESTIGACIONES PREVIAS}

Este apartado presenta brevemente investigaciones previas realizadas en algunas de las áreas temáticas que sustentan este estudio.

Investigaciones en CMC realizadas por Meunier (1996) y Warschauer (1995/1996) en relación con la interacción han mostrado que las diferencias individuales de los 
estudiantes (género, motivación, independencia, dependencia) disminuyen en comparación con la comunicación cara a cara. Por otra parte, en investigaciones efectuadas por Kelm (1992) y Kern (1995) se hace evidente el enfoque en el significado en tareas CMC si éstas desarrollan un tema compartido por ambos estudiantes durante la conversación.

Liontas (2001) condujo un estudio experimental pre-post test en el cual los estudiantes del grupo experimental obtuvieron un logro significativo en comparación con el grupo control, en la habilidad comunicativa, al introducir el uso de tecnologías (TICs) en el proceso de enseñanza aprendizaje de L2. En un estudio que incorporó el uso de e-mail y el enfoque basado en tareas utilizando la Web, realizado por Terry (2000), se obtuvo como resultado un aumento en la motivación de los estudiantes, además de proveer práctica adicional fuera de la sala de clases a través del uso de correo electrónico.

En un estudio experimental en el uso de e-mail y enfoque por tareas para la enseñanza del ruso, realizado por Samsonov y Shih (2000), los estudiantes del grupo experimental mostraron un mayor desarrollo comunicacional que los estudiantes del grupo control. Este mismo estudio mostró que uno de los factores que más influyó en la actuación de los alumnos fue la motivación (Krashen 1985). Del mismo modo, Morales (2008) propone un modelo de aprendizaje combinado (presencial y no presencial), que incorpora el enfoque por tareas, el aprendizaje cooperativo y CMC (chat, e-mail y weblog), para la enseñanza de inglés como lengua extranjera, el cual concluyó que en el caso del grupo experimental la habilidad que se vio más favorecida fue la producción escrita, seguida de la producción oral y comprensión escrita, mientras que el grupo control mostró un mayor aumento en la comprensión auditiva, seguida por la producción y comprensión escrita, relegando a la producción oral al último lugar.

A partir de estas investigaciones es posible concluir que:

- El uso CMC reduciría las diferencias individuales.

- En las tareas CMC primaría el enfoque en el significado (negociación de significado).

- La inclusión de tecnologías en la sala de clases beneficiaría las habilidades lingüísticas de los estudiantes.

- El uso de e-mail y del enfoque por tareas promovería la motivación en los alumnos.

\section{ESTUDIO CUASIEXPERIMENTAL}

Con el fin de determinar si la aplicación de los principios metodológicos del enfoque basado en tareas, el aprendizaje cooperativo y la metodología CALL utilizados de manera combinada en el diseño de actividades es eficaz para el desarrollo de las habilidades lingüísticas, se explora evidencia empírica acerca de la efectividad de esta metodología en el aprendizaje de inglés como lengua extranjera en un estudio cuasiexperimental pre- y post-test grupo control para responder la pregunta de investigación que orienta el análisis de los datos: ¿mejorará el aprendizaje del inglés como lengua extranjera en los alumnos que se vean expuestos al modelo metodológico propuesto? Para responder dicha pregunta de investigación se formuló la siguiente hipótesis. 


\subsection{HIPOTESIS}

La habilidad comunicativa de los estudiantes en temáticas específicas puede ser apoyada de manera efectiva a través de una metodología CALL fundamentada en losenfoques por tareas y cooperativo.

\subsection{METODOLOGIA}

\subsubsection{Muestra}

La muestra utilizada en este estudio cuasiexperimental está compuesta por 12 alumnos pertenecientes a un instituto de lenguas de la ciudad de Concepción inscritos en el curso Adultos Regular 1. Este curso comprende 3 horas semanales, divididas en 2 sesiones de 1,5 horas cada una. El rango de edad de los estudiantes fluctúa entre los 23 y 35 años. Del universo total de la muestra, el $58 \%$ era de sexo femenino y el $42 \%$ de sexo masculino. El nivel de competencia lingüística de entrada del 100\% de los alumnos es de nivel básico, evaluado sobre la base de una prueba de admisión de conocimientos específicos que realiza la institución. Para efectos de la investigación, se determinó que los alumnos del curso 1 (lunes y miércoles) integran al grupo experimental y los estudiantes del curso 2 (martes y jueves) al grupo control. Esta distribución se realizó en función de la disponibilidad de horario de los estudiantes dispuesta por la institución académica.

Grupo Experimental:

Los estudiantes de este grupo, después del pretest, trabajaron con los contenidos sobre la base del módulo de intervención lingüística. Dicho módulo presentaba una metodología presencial mediatizada por la tecnología para el desarrollo de las habilidades lingüísticas sustentada en el enfoque por tareas y el enfoque cooperativo. Las clases se realizaron en aula y en un laboratorio de computación. Durante el desarrollo del módulo los estudiantes utilizaron un manual y un weblog, los cuales sirvieron de soporte para trabajar focalización en la forma, a la vez de proveerles práctica en las cuatro habilidades. A su vez, los estudiantes utilizaron las tecnologías de información y comunicación (TICs) para la consecución de sus tareas finales. Al final del proceso se les aplico un postest.

\section{Grupo Control:}

Los estudiantes de este grupo después del pretest trabajaron con los contenidos sobre la base de la modalidad presencial (siempre en aula) sustentada en el enfoque comunicativo. En esta investigación se describe como metodología comunicativa a la instrucción utilizando el texto y CD de audio obligatorio para el curso. Ambos grupos trabajaron con los mismos contenidos y objetivos. Al final del proceso se les aplicó un postest.

\subsubsection{Procedimiento}

Para medir el proceso de intervención lingüística, ambos grupos se sometieron a dos evaluaciones. Con el propósito de resguardar el nivel de proficiencia de los alumnos y verificar que el proceso de intervención estuviera acorde a la habilidad comunicativa de 
los estudiantes se utiliza el Cambridge Key English Test (KET) como pretest y luego como postest. Y, además, se utiliza un pre y postest de proceso de intervención lingüística que permite evaluar los contenidos incluidos en dicho proceso.

\subsection{PROCESO DE INTERVENCION LINGÜISTICA}

El proceso de intervención linguiística ha sido diseñado sobre la base de una metodología mixta que incluye la enseñanza basada en tareas y el enfoque cooperativo mediatizado por la tecnología a través del uso de Tics, e-mail, y weblog y tiene como objetivo: promover el trabajo cooperativo utilizando tareas que sirvan de apoyo en el proceso de aprendizaje de inglés como lengua extranjera y utilizar los recursos tecnológicos presentes en la web en beneficio del aprendizaje de lenguas.

Este módulo de intervención lingüística fue integrado en el programa de la asignatura. Por lo cual, los tópicos, metas comunicativas y estructuras gramaticales que se trabajaron en el modelo se consideraron de acuerdo a los contenidos incluidos en el curso Adultos Regular 1. Para la práctica de las habilidades y formas lingüísticas se diseñó un manual, y un weblog sobre la base de las tecnologías e información y comunicación asincrónicas (blog y correo electrónico).

El tiempo de duración del módulo fue de 14 sesiones, divididas en clases en aula (9 sesiones) y clases en laboratorio (5 sesiones). La Tabla 1 presenta un resumen del procedimiento del modelo metodológico que utilizó el grupo experimental.

\section{Tabla 1}

Distribución del Modelo de Intervención Linguiística

\begin{tabular}{|l|l|}
\hline Introducción al módulo de intervención lingüística. & 1 sesión. \\
\hline Clases en aula. & 8 sesiones. \\
\hline Clases en laboratorio. & 4 sesiones. \\
\hline Evaluación. (Tareas finales) & 1 sesión. \\
\hline
\end{tabular}

\subsubsection{Manual}

Se diseñó un manual de trabajo para los estudiantes de acuerdo a los postulados de la enseñanza basada en tareas propuestas por Skehan (1996), Willis (1996) y Ellis (2003), y del enfoque cooperativo de Slavin (1990), Fathman y Kessler (1993) y Trujillo (2007) siguiendo el modelo para la creación de unidades didácticas de Estaire (2004). En este manual los alumnos trabajaron en cuatro módulos con distintas temáticas, distintas formas lingüísticas y distinto léxico.

El tipo de tareas incluidas en el manual fue diverso y la mayor parte de las tareas buscaba que el estudiante resolviera problemas, compartiera sus experiencias personales y creara un producto a partir del análisis de información. En relación a las técnicas cooperativas que se utilizaron en esta investigación se pueden mencionar aprendiendo juntos grupo de investigación y jigsaw. El manual incluyó tareas para el desarrollo de las cuatro habilidades lingüísticas más un componente de enfoque en la forma. 


\subsubsection{Weblog}

Se desarrolló e implementó un weblog como herramienta de aprendizaje en el contexto de las metodologías CALL, por tareas y cooperativo. Este weblog (www.englishvirtualclasses. blogspot.com) fue diseñado utilizando el sistema online gratuito Blogger, para complementar y fortalecer el trabajo realizado en el aula tanto dentro como fuera del horario de clases.

Los alumnos utilizaban este recurso para la ejercitación de sus habilidades lingüísticas, la comprensión oral se reforzó a través de ejercicios de comprensión auditiva y videos. La comprensión lectora se reforzó a partir del trabajo en las tareas finales a partir de la búsqueda de información en páginas web indicadas por el profesor cuyos links se incluyeron en el weblog. Mientras que la producción escrita se reforzó a partir de los comentarios que los alumnos hacían en el weblog. Además del trabajo en las cuatro habilidades lingüísticas, el weblog incluyeron ejercitación para el léxico y el enfoque en la forma. Para amenizar un poco el trabajo se incluyó juegos en cada uno de los módulos. En la Figura 1 se observa la página principal del blog de la clase que contiene una visión panorámica y que le permite al alumno poder desplazarse a través de los cuatro módulos.

Como se observa en la Figura 2, la práctica de la comprensión auditiva también se vio apoyada en el weblog. Se insertaron videos descargados desde el sitio gratuito www.videojug.com para ilustrar situaciones de comunicación real. En esta tarea, los alumnos deben observar el video e identificar los cuatro remedios caseros para la acidez estomacal. Luego, deben responder algunas preguntas.

Para incrementar el léxico de los estudiantes se incluyen actividades de vocabulario. La Figura 3 ejemplifica una actividad de léxico en la cual los estudiantes deben asociar la imagen con el problema de salud.

El enfoque en la forma también se incorpora en el weblog. La Figura 4 ilustra una tarea de enfoque en la forma en la cual el alumno debe completar un texto sobre la ciudad de Sao Paulo, seleccionando las formas comparativas y superlativas correctas.

Figura 1

Imagen de la pantalla principal del weblog

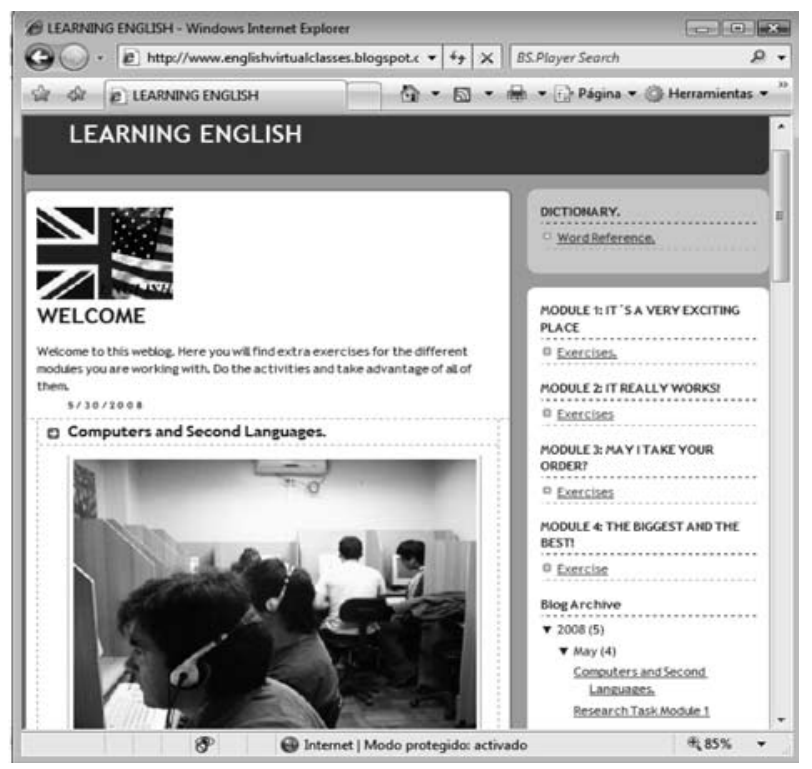


Figura 2

Ejemplo de práctica de la comprensión auditiva a través del weblog

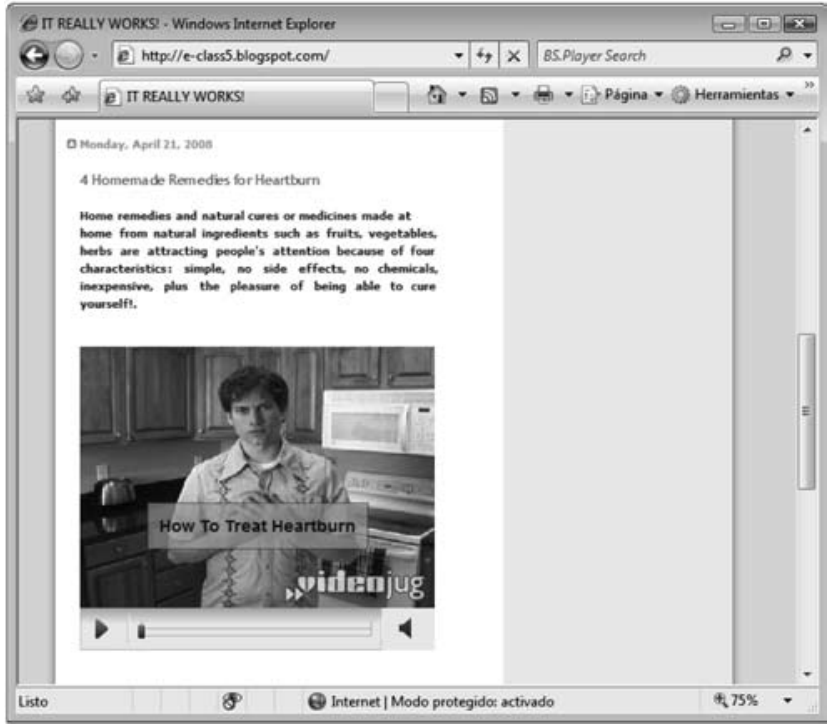

Figura 3

Ejemplo de actividad de vocabulario

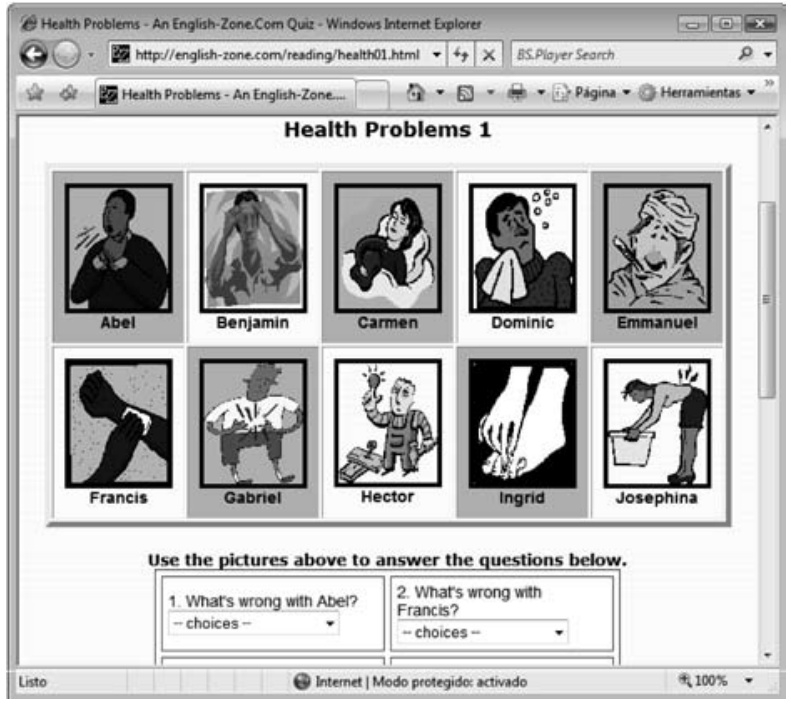




\section{Figura 4}

Ejemplo de actividad de enfoque en la forma

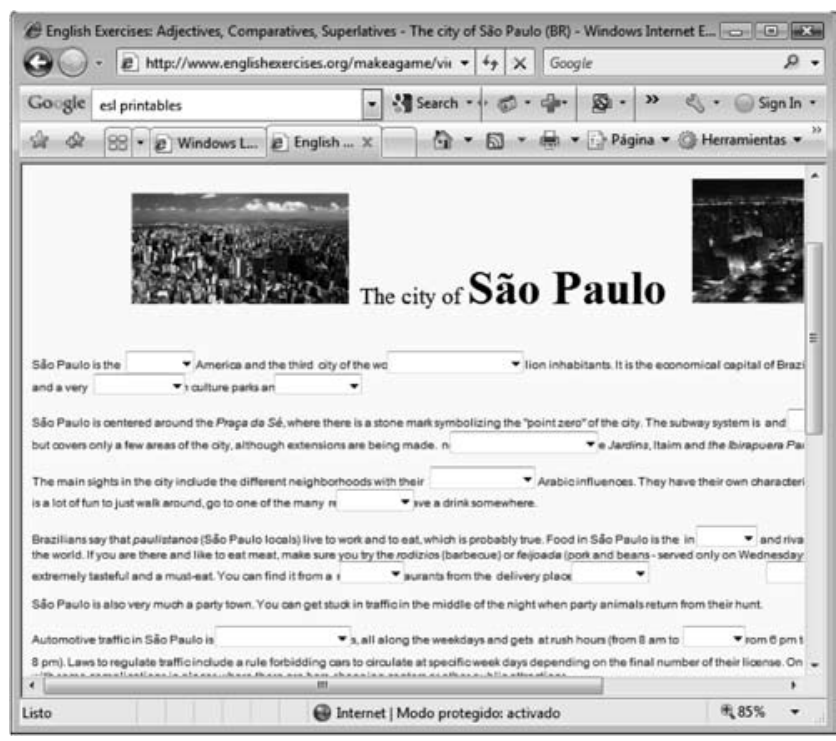

Como se muestra en la Figura 5 los estudiantes practicaron la producción escrita en el weblog a través de distintas tareas asignadas durante el proceso. La finalidad de esta tarea en forma particular era decidir (como grupo) un lugar para visitar, como clase, una vez finalizado el curso.

\section{Figura 5}

Ejemplo de comentarios sobre una tarea

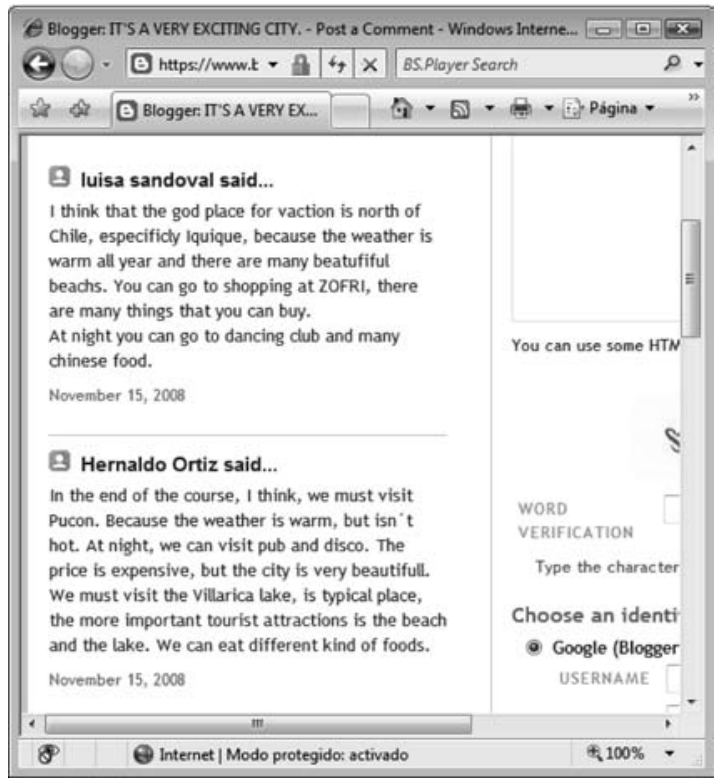




\section{ANALISIS DE LOS RESULTADOS}

Una vez finalizado el ciclo de exposición al módulo de intervención lingüística del grupo experimental y las clases tradicionales del grupo control, se procedió a corregir el pretest y postest de los 12 sujetos que componían la muestra.

\section{- Resultados del Test KET:}

En relación con la hipótesis planteada en la investigación, la Tabla 2 presenta los resultados obtenidos en el pretest y postest KET del grupo experimental. Se ilustran los resultados que cada sujeto (S) de este grupo obtuvo por habilidad. Es posible observar que la habilidad que se vio más favorecida fue la producción oral, con un $45 \%$ de aumento. La producción escrita y la comprensión auditiva aumentaron un $35 \%$ y un $24 \%$, respectivamente, y la comprensión lectora sólo lo hizo un $20 \%$.

La Tabla 3 muestra los resultados observados en el pretest y postest del Grupo Control. A partir de esta tabla es posible observar que la habilidad que logró un mayor incremento fue la producción oral, con una diferencia de un $37 \%$. Luego le sigue la producción escrita con un $25 \%$ y la comprensión lectora con un $20 \%$ y finalmente la comprensión auditiva con un $12 \%$.

La Tabla 4 presenta la diferencia del resultado final de los grupos tomando en cuenta los porcentajes finales de los test. Como se puede apreciar, el progreso en el aprendizaje obtenido por el grupo experimental fue mayor que el logrado por el grupo control.

\section{- Resultados de Test de Proceso de Intervención Lingüística:}

En relación con el test de proceso de intervención lingüística, la Tabla 5 presenta los resultados obtenidos en el pretest y postest del grupo experimental.

\section{Tabla 2}

Resultados Pre-Post test grupo experimental

\begin{tabular}{|c|c|c|c|c|c|c|c|c|}
\hline S & \multicolumn{2}{|c|}{$\begin{array}{c}\text { Comprensión } \\
\text { Lectora } \\
\%\end{array}$} & \multicolumn{2}{c|}{$\begin{array}{c}\text { Producción } \\
\text { Escrita } \\
\%\end{array}$} & \multicolumn{2}{c|}{$\begin{array}{c}\text { Comprensión } \\
\text { Auditiva } \\
\%\end{array}$} & \multicolumn{2}{c|}{$\begin{array}{c}\text { Producción } \\
\text { Oral } \\
\%\end{array}$} \\
\hline 1 & 40 & 71 & 50 & 85 & 64 & 92 & 33 & 78 \\
\hline 2 & 43 & 66 & 60 & 90 & 72 & 96 & 55 & 100 \\
\hline 3 & 46 & 66 & 50 & 80 & 68 & 92 & 44 & 90 \\
\hline 4 & 49 & 66 & 55 & 90 & 64 & 88 & 33 & 78 \\
\hline 5 & 34 & 46 & 50 & 85 & 60 & 84 & 22 & 67 \\
\hline 6 & 34 & 51 & 40 & 75 & 60 & 80 & 33 & 56 \\
\hline & Pre & Post & Pre & Post & Pre & Post & Pre & Post \\
\hline
\end{tabular}


Tabla 3

Resultados Pre-Post test grupo control

\begin{tabular}{|c|c|c|c|c|c|c|c|c|}
\hline S & \multicolumn{2}{|c|}{$\begin{array}{c}\text { Comprensión } \\
\text { Lectora } \\
\%\end{array}$} & \multicolumn{2}{c|}{$\begin{array}{c}\text { Producción } \\
\text { Escrita } \\
\%\end{array}$} & \multicolumn{2}{c|}{$\begin{array}{c}\text { Comprensión } \\
\text { Auditiva } \\
\%\end{array}$} & \multicolumn{2}{c|}{$\begin{array}{c}\text { Producción } \\
\text { Oral } \\
\%\end{array}$} \\
\hline 1 & 43 & 60 & 50 & 56 & 76 & 88 & 33 & 67 \\
\hline 2 & 40 & 54 & 40 & 52 & 64 & 80 & 33 & 67 \\
\hline 3 & 43 & 57 & 35 & 52 & 72 & 84 & 33 & 56 \\
\hline 4 & 49 & 60 & 50 & 60 & 68 & 80 & 44 & 89 \\
\hline 5 & 46 & 57 & 55 & 64 & 60 & 76 & 44 & 67 \\
\hline 6 & 54 & 77 & 65 & 68 & 72 & 88 & 56 & 89 \\
\hline & Pre & Post & Pre & Post & Pre & Post & Pre & Post \\
\hline
\end{tabular}

Tabla 4

Diferencia final entre grupo experimental y grupo control test KET

\begin{tabular}{|l|c|c|c|}
\hline \multicolumn{1}{|c|}{ Grupo } & Pretest & Postest & Diferencia \\
\hline Experimental & $49 \%$ & $75 \%$ & $26 \%$ \\
\hline Control & $52 \%$ & $70 \%$ & $18 \%$ \\
\hline
\end{tabular}

\section{Tabla 5}

Resultados Pre-Post test grupo experimental

\begin{tabular}{|c|l|l|l|l|l|l|l|l|l|l|l|l|}
\hline S & $\begin{array}{c}\text { Comprensión } \\
\text { Auditiva } \\
\%\end{array}$ & \multicolumn{2}{|c|}{$\begin{array}{c}\text { Gramática } \\
\%\end{array}$} & \multicolumn{2}{|c|}{$\begin{array}{c}\text { Vocabulario } \\
\%\end{array}$} & \multicolumn{2}{c|}{$\begin{array}{c}\text { Producción } \\
\text { Escrita } \\
\%\end{array}$} & $\begin{array}{c}\text { Comprensión } \\
\text { Lectora } \\
\%\end{array}$ & \multicolumn{2}{|c|}{$\begin{array}{c}\text { Producción } \\
\text { Oral } \\
\%\end{array}$} \\
\hline 1 & 50 & 87,5 & 37,5 & 87,5 & 25 & 100 & 50 & 83 & 33,3 & 66,6 & 33,3 & 66,6 \\
\hline 2 & 62,5 & 87,5 & 50 & 87,5 & 75 & 100 & 66,6 & 100 & 66,6 & 66,6 & 55,5 & 100 \\
\hline 3 & 50 & 75 & 37,5 & 62,5 & 25 & 75 & 66,6 & 66,6 & 66,6 & 66,6 & 44,4 & 88,8 \\
\hline 4 & 50 & 75 & 37,5 & 62,5 & 50 & 100 & 50 & 83 & 66,6 & 66,6 & 33,3 & 77,7 \\
\hline 5 & 50 & 87,5 & 37,5 & 75 & 50 & 100 & 50 & 91,6 & 33,3 & 66,6 & 33,3 & 66,6 \\
\hline 6 & 50 & 75 & 25 & 75 & 0 & 50 & 25 & 66,6 & 66,6 & 66,6 & 33,3 & 55,5 \\
\hline & Pre & Post & Pre & Post & Pre & Post & Pre & Post & Pre & Post & Pre & Post \\
\hline
\end{tabular}

Como se puede observar a partir de la Tabla 6, la habilidad que se vio más favorecida fue la producción oral, con un $37 \%$ de aumento. La producción escrita y la comprensión 
auditiva aumentaron un $30,5 \%$ y un $29,2 \%$ respectivamente, y la comprensión lectora sólo lo hizo un 11,1 .

La Tabla 6 muestra los resultados observados en el pretest y postest del Grupo Control. De acuerdo a estos datos, la habilidad que logró un mayor incremento fue la producción oral, con una diferencia de un 33,3\%. Luego le siguen la producción escrita con un $17,9 \%$ y la comprensión auditiva con un $17,4 \%$ y finalmente la comprensión lectora con un $14 \%$.

Tabla 6

Resultados Pre-Post test grupo control

\begin{tabular}{|c|c|c|c|c|c|c|c|c|c|c|c|c|}
\hline S & $\begin{array}{c}\text { Comprensión } \\
\text { Auditiva } \\
\%\end{array}$ & \multicolumn{2}{|c|}{$\begin{array}{c}\text { Gramática } \\
\%\end{array}$} & \multicolumn{2}{|c|}{$\begin{array}{c}\text { Vocabulario } \\
\%\end{array}$} & \multicolumn{2}{|c|}{$\begin{array}{c}\text { Producción } \\
\text { Escrita } \\
\%\end{array}$} & $\begin{array}{c}\text { Comprensión } \\
\text { Lectora } \\
\%\end{array}$ & \multicolumn{2}{c|}{$\begin{array}{c}\text { Producción } \\
\text { Oral } \\
\%\end{array}$} \\
\hline 1 & 62,5 & 87,5 & 50 & 75 & 25 & 75 & 50 & 66,6 & 50 & 66,6 & 33,3 & 66,6 \\
\hline 2 & 50 & 75 & 25 & 50 & 50 & 100 & 58,3 & 75 & 50 & 66,6 & 33,3 & 66,6 \\
\hline 3 & 75 & 87,5 & 12,5 & 50 & 25 & 50 & 33,3 & 50 & 83 & 100 & 33,3 & 55,5 \\
\hline 4 & 75 & 100 & 37,5 & 75 & 50 & 50 & 66,6 & 83 & 66,6 & 66,6 & 55,5 & 88,8 \\
\hline 5 & 50 & 62,5 & 12,5 & 62,5 & 50 & 75 & 66,6 & 83 & 66,6 & 100 & 33,3 & 77,7 \\
\hline 6 & 75 & 75 & 12,5 & 62,5 & 75 & 100 & 66,6 & 91,6 & 100 & 100 & 55,5 & 88,8 \\
\hline & Pre & Post & Pre & Post & Pre & Post & Pre & Post & Pre & Post & Pre & Post \\
\hline
\end{tabular}

La Tabla 7 presenta la diferencia del resultado final de los grupos tomando en cuenta los porcentajes finales de los test. Como se puede apreciar, el progreso en el aprendizaje obtenido por el grupo experimental fue mayor que el logrado por el grupo control.

Tabla 7

Diferencia final entre grupo experimental y grupo control en test de intervención lingüística

\begin{tabular}{|l|c|c|c|}
\hline \multicolumn{1}{|c|}{ Grupo } & Pretest & Postest & Diferencia \\
\hline Experimental & $46 \%$ & $78 \%$ & $32 \%$ \\
\hline Control & $52 \%$ & $75 \%$ & $23 \%$ \\
\hline
\end{tabular}

Al considerar las diferencias obtenidas en ambos entre el grupo experimental y el grupo control en el test KET y el test del proceso de intervención lingüística se puede afirmar que el aprendizaje ganado por el grupo experimental se debió a la organización del diseño, la forma en que fueron presentados los materiales y las vías de entrega de éstos (utilizando recursos de internet). Todo esto favoreció que los alumnos desarrollaran 
las habilidades de forma integrada y compacta involucrándolos de una manera profunda a los objetivos del modelo.

Con el objetivo de comprobar si el mejoramiento en el aprendizaje del grupo experimental presenta validez estadística, se realizaron pruebas estadísticas para el test de habilidad comunicativa y para el test de proceso de intervención lingüística.

\section{Análisis estadístico de test de KET:}

Para comprobar la efectividad del modelo de intervención lingüística se compararon los resultados del pre y postest en cada uno de los grupos estudiados. Al aplicar una prueba estadística para la diferencia de medias de ambos tests se obtiene, en el caso del grupo experimental, que hay diferencias estadísticas significativas $(\mathrm{p}=0,0003)$. En el caso del grupo control, las diferencias estadísticas también son significativas $(p=0,0008)$. Si bien los resultados muestran diferencias significativas en ambos grupos, lo relevante es que en el caso del grupo experimental hubo un mayor incremento que en el grupo control.

Luego, al comparar las diferencias de los resultados de los pre y postest entre los grupos experimental y de control, mediante un test de comparación de medias se obtuvo un valor $(\mathrm{p}=0,05)$, lo que no permite concluir con certeza sobre la diferencia de medias entre los grupos; sin embargo, dicho resultado puede ser atribuido al escaso tamaño de muestra.

\section{Análisis estadístico de test de Proceso de intervención lingüística:}

El análisis estadístico del proceso de intervención lingüística se realizó del mismo modo que el análisis de habilidad comunicativa.

Al aplicar una prueba estadística para la diferencia de medias de ambos test se obtiene, en el caso del grupo experimental, que hay diferencias estadísticas significativas $(\mathrm{p}<0,0001)$ entre ambos test. En el caso del grupo control, las diferencias estadísticas también son significativas $(\mathrm{p}=0,0003)$.

Luego, comparar las diferencias de los resultados de los pre y postest entre los grupos experimental y de control a partir de un test de comparación de medias se encontró que hay evidencia estadística significativa $(\mathrm{p}=0,003)$, para concluir que las medias de las diferencias de ambos grupos son distintas, presentando un valor mayor en el caso del grupo intervenido.

\section{CONCLUSIONES}

Este artículo se ha centrado en proponer e implementar un modelo metodológico efectivo en el desarrollo de las habilidades lingüísticas en inglés como lengua extranjera sobre la base del enfoque basado en tareas, el enfoque cooperativo y la metodología CALL, los cuales a la luz del marco teórico propuesto han probado ser efectivos en el proceso de enseñanza-aprendizaje de una segunda lengua.

Los resultados obtenidos en este estudio evidencian que las habilidades lingüísticas de los estudiantes se vieron favorecidas a partir de la metodología propuesta en esta in- 
vestigación. Los resultados señalan que las habilidades de producción oral y producción escrita fueron las más beneficiadas con el modelo metodológico propuesto. La producción oral se vio beneficiada por el uso de tareas de comunicación y del trabajo cooperativo que permitió una mayor independencia de los alumnos en relación al docente y un mayor intercambio en la lengua meta. El incremento en la oralidad también podría deberse al enfoque en la negociación de significado por sobre el enfoque en la forma durante el desarrollo de las tareas de comunicación.

La producción escrita, por su parte, se trabajó en el manual del estudiante en tareas tendientes al desarrollo de la escritura en forma particular, así también, el enfoque cooperativo requirió que los alumnos tomaran notas para luego poder compartirlas con el resto de la clase. Del mismo modo, la escritura se vio favorecida a través del uso del weblog y del correo electrónico.

Estas dos habilidades (producción oral y escrita) fueron las que se beneficiaron en mayor manera pero tanto el manual como el weblog tuvieron como objetivo la integración y el desarrollo de las cuatro habilidades linguiísticas a partir de las tareas que debieron realizar los estudiantes.

Luego de examinar los resultados obtenidos es posible esbozar algunas conclusiones:

- Los modelos metodológicos de enseñanza basada en tareas y el aprendizaje cooperativo potenciaron el trabajo en el aula y las oportunidades de interacción en la lengua meta.

- En relación con el enfoque basado en tareas, es posible señalar que el uso de tareas comunicativas permitió que los estudiantes compartieran experiencias personales y sus puntos de vista frente a las tareas de resolución de problemas, lo cual motivó la participación y el uso de la lengua con fines comunicativos reales.

- En cuanto al aprendizaje cooperativo, el uso de grupos de trabajo en los cuales cada miembro cumpliera un rol determinado para el logro de una meta común mostró ser una alternativa beneficiosa en el desarrollo de las tareas; del mismo modo, esta modalidad de trabajo creó un clima de respeto y compañerismo, así como también una interdependencia e interés de que todos los miembros del grupo aprendieran y contribuyeran en la resolución de las tareas asignadas.

- El uso de las Tecnologías de Información y Comunicación tuvo un rol fundamental, ya que permitió por una parte el acceso a un mayor input para los estudiantes y una exposición a la lengua meta en un contexto real (páginas web).

- El uso de Weblog proveyó a los estudiantes práctica adicional en comprensión lectora, comprensión auditiva y producción escrita; promovió la exploración de sitios web y a la vez promovió el intercambio verbal online.

Mediante esta investigación fue posible llegar a conclusiones que pretenden contribuir al proceso de enseñanza y aprendizaje de lenguas. Estos resultados no pretenden ser concluyentes dado el tamaño de la muestra, pero sí evidencian una tendencia que señalaría que sería ventajoso replicar la metodología propuesta en esta investigación. 


\section{BIBLIOGRAFIA}

Bygate, M., Skehan, P. \& Swain, M. (eds.). (2001). Researching pedagogic tasks. New York: Pearson.

Cameron, K. (ed.). (1999). CALL: Media, Design \& Applications. The Netherlands: Swets \& Zeitlinger Publishers.

Estaire, S. (2004). "La programación de unidades didácticas a través de tareas". Revista Electrónica de Didáctica ELE, I.

Johnson, D. \& Johnson, R. (1987). Learning together and alone. New Jersey. Prentice Hall: Englewood Cliffs.

Johnson, D. \& Johnson, R. (1998). "Effective staff development in cooperative learning: Training, transfer, and long-term use". En C. Brody y N. Davidson (Eds.). Professional development for cooperative learning: Issues and approaches. Albany: State University of New York Press, pp. 223-242.

Kelm, O. (1992). The use of synchronous computer networks in second language instruction: A preliminary report. Foreign Language Annals, 25 (5), 441-454.

Kern, R. (1995). Restructuring classroom interaction with networked computers: Effects on quantity and characteristics of language production. Modern Language Journal, 79, 457-476.

Liontas, J. (2001). "Reading and Multimedia annotations: going beyond bells and whistles, hot links and pop-up windows" en The IALL journal of language learning technologies, vol. 33 $\mathrm{N}^{\mathrm{o}}$ 1, pp. 53-78.

Long, M. H. (1985). A role for instruction in second language acquisition: Task-based language training. In K. Hyltenstam and M. Pienemann (Eds.), Modelling and assessing second language acquisition (pp. 77-99). Clevedon: Multilingual Matters.

Meunier, L. E. (1996). Personality differences and motivational factors in distance learning. In F. Borchardt, C. Bradin, and E. Johnson (Eds.), Distance learning, Proceedings of the Computer Assisted Language Instruction Consortium 1996 Annual Symposium (pp. 181-188). Albuquerque, NM: The University of New Mexico.

Morales, S. (2008). La efectividad de un modelo de aprendizaje combinado para la enseñanza de inglés como lengua extranjera. Revista de Lingüística Teórica y Aplicada, 46 (2), (pp. 95-118).

Nunan, D. (1989) Designing Tasks for the Communicative Classrooms. Cambridge: Cambridge University Press.

Pica, T., Kanagy, R. \& Falodun, J. (1993). Choosing and using communication tasks for second language instruction. In G. Crookes and S. Gass (Eds.), Tasks and language learning: Integrating theory \& practice. Clevedon, England: Multilingual Matters, Ltd.

Prabhu, N. S. (1987). Second Language Pedagogy. Oxford: Oxford University Press.

Richards, J. \& Rodgers, T. 2001. Approaches and Methods in Language Teaching. Cambridge: Cambridge University Press.

Samsonov, P. \& Shih D. 2000. "Experimental teaching of Russian using email communication", en The IALL journal of language learning technologies, vol. 32, $\mathrm{N}^{\circ} 2$, pp. 15-22.

Sharan, Y. \& Sharan, S. (1992) Expanding cooperative learning through group investigation. New York: Teachers College Press.

Skehan, P. (1996). "A framework for Implementing Task-Based Instruction". Applied Linguistics, $17,38-62$.

Slavin, R. E. (1990): Cooperative learning: Theory, research and practice. Massachusetts: Allyn and Bacon.

Terry, Robert (2000). "You've got mail! It's time to grade" en The IALL journal of language learning technologies, vol. 33, $\mathrm{N}^{\circ} 1$, pp. 79-98. 
Walters, L. (2000). "Four Leading Models", Harvard Education Letter's Research Online. Disponible en http://www.edletter.org/past/issues/2000-mj/models.shtml

Warschauer, M. (1995). E-mail for English teaching. Alexandria, VA: TESOL Publications.

Warschauer, M. (1996). Virtual connections: On-line activities \& projects for networking language learners. Honolulu, HI: Second Language Teaching \& Curriculum Center, University of Hawai'i.

Willis, J. (1996). A framework forTask-Based Learning. London: Longman.

Willis, J. y D. Willis (2007). Doing task-based teaching. Oxford: Oxford University Press. 
